\title{
Dissipative squeezed vacuum in non-equilibrium thermo field dynamics
}

\author{
Kyo Yoshida ${ }^{1 *}$, Tsuyoshi Hayashi ${ }^{1 \dagger}$, Sachiko Kitajima ${ }^{2}$ \\ and Toshihico Arimitsu ${ }^{1}$ \\ ${ }^{1}$ Graduate School of Pure and Applied Sciences, University of Tsukuba \\ Ibaraki 305-8571, Japan \\ 2 Graduate School of Humanities and Sciences, Ochanomizu University \\ Tokyo 112-8610, Japan
}

March 11, 2010

Preprint of Physica A 389(4), 705-722 (2010).

\begin{abstract}
The degenerate parametric amplification accompanied by dissipation is analyzed within the canonical operator formalism for quantum dissipative systems named non-equilibrium thermo field dynamics. The vacuum of the system is subject to both dissipation and breaking of phase symmetry due to squeezing. The annihilation-creation operators for the vacuum are derived and the structure of the vacuum is examined. The effects of dissipation on squeezing and uncertainty relation are estimated.
\end{abstract}

\section{Introduction}

Squeezed states in quantum optics have a wide scope of applications including quantum information technology for continuous variables [1]. Squeezed states can be generated by nonlinear optical process such as degenerate parametric amplification. Note that, during the practical generating process of the squeezed states, the system inevitably experiences dissipation due to the interaction with environmental noises. Therefore, it is important to know the effect of dissipation on the generation of the squeezed states.

Conventionally, dissipation in quantum systems is treated within the density operator method. The quantum master equation of the system of interest is obtained by projecting out the reservoir by means of the damping theory [2] from the quantum Liouville equation for the total system, i.e., the system of

*e-mail: yoshida.kyo.fu@u.tsukuba.ac.jp

†Present Address: M.C.C., 1-4-1 Koishikawa, Bunkyo-ku, Tokyo 112-0002, Japan 
interest weakly and linearly coupled to the reservoir. The resulting equation is represented in a form of complex entanglement of operators. Usually, the entanglement is dissolved by mapping the operator equation into a partial differential equation in a certain c-number function space [3]. The system of degenerate parametric amplifier attached to a heat reservoir was analyzed by mapping the density operator to the Wigner distribution function [4].

Non-Equilibrium Thermo Field Dynamics (NETFD) [5, 6, 7] is a canonical operator formalism which provides us with a method to treat dissipative quantum systems in a similar manner as usual quantum field theory (QFT), i.e., in terms of the operator algebra and the representation space. The representation space of NETFD, named thermal space, is composed of a direct product of two Hilbert spaces. Two kinds of operators, non-tilde operators and tilde operators, acting on each of the Hilbert spaces are introduced. Introduction of the two kinds of operators results in reducing the quantum master equation, within the framework of NETFD, into the form of the Schrödinger equation (in the unit $\hbar=1)$

$$
\frac{\partial}{\partial t}|0(t)\rangle=-\mathrm{i} \hat{H}|0(t)\rangle,
$$

where $|0(t)\rangle$, called thermal ket-vacuum, is an element of the thermal space and the infinitesimal time-evolution generator $\hat{H}$, called hat-Hamiltonian, consists of both non-tilde and tilde operators. We call (1.1) the dissipative Schrödinger equation. Note that the thermal ket-vacuum $|0(t)\rangle$ is unstable and depends on time $t$. The time dependence of the vacuum is an unique features in NETFD and the situation is quite different from the cases in usual QFT. One can introduce the time-dependent annihilation operator $\gamma_{t}\left(\tilde{\gamma}_{t}\right)$ which is specified by $\gamma_{t}|0(t)\rangle=$ $0\left(\tilde{\gamma}_{t}|0(t)\rangle=0\right)$ and its canonical conjugate $\gamma^{q}\left(\tilde{\gamma}^{q}\right)$. The operators $\gamma^{q}$ and $\tilde{\gamma}^{q}$ are time-independent reflecting the fact that the thermal bra-vacuum $\langle 1|$ is timeindependent (see Sec. 2). Operator algebra such as normal ordering with respect to $\gamma_{t}, \tilde{\gamma}_{t}, \gamma^{q}$ and $\tilde{\gamma}^{q}$ can be manipulated in a similar way as in usual QFT.

In this paper, we apply NETFD to the degenerate parametric amplification with dissipation (see Ref. [8] for the preliminary report). The formalism of NETFD is briefly summarized in Sec. 2. We introduce annihilation-creation operators for the vacuum of the semi-free field, i.e. the field subject purely to dissipation and free from squeezing, in Sec. 3. The model, i.e., hat-Hamiltonian, of the degenerate parametric amplification with the dissipation, is specified in Sec. 4. Then, we derive the annihilation-creation operators of the vacuum corresponding to the model, i.e., the dissipative squeezed vacuum, in Sec. 5. We introduce the quadrature-phase amplitudes in Sec. 6, and they are related to the structure of the dissipative squeezed vacuum in Sec. 7. We summarize the implication of the present study on the effect of dissipation on squeezing and uncertainty relation in Sec. 8. The discussion is given in Sec. 9. "The principle of correspondence" which relates the density operator method and NETFD is given in Appendix A. In the analysis, we will use the vacuum in the interaction representation. The relation between the Schrödinger and interaction representations is given in Appendix B. For comparison with the present analysis, 
we show an alternative analysis based on mapping the dissipative Schrödinger equation to a differential equation of a c-number function in Appendix C.

\section{Formalism of NETFD}

In this section, we briefly review the formalism of NETFD. ${ }^{1}$ Throughout this paper, we restrict ourselves to bosonic systems. ${ }^{2}$

Within the formalism of NETFD, any operator $A$ is accompanied by its tilde conjugate $\tilde{A}$. The operators $A$ and $\tilde{A}$ are called, respectively, non-tilde operator and tilde operator. ${ }^{3}$ The tilde conjugation is defined by the following rules [12],

$$
\begin{aligned}
\left(A_{1} A_{2}\right)^{\sim} & =\tilde{A}_{1} \tilde{A}_{2}, \\
\left(c_{1} A_{1}+c_{2} A_{2}\right)^{\sim} & =c_{1}^{*} \tilde{A}_{1}+c_{2}^{*} \tilde{A}_{2}, \\
(\tilde{A})^{\sim} & =A, \\
\left(A^{\dagger}\right)^{\sim} & =\tilde{A}^{\dagger},
\end{aligned}
$$

where $A, A_{1}$ and $A_{2}$ represent operators, and $c_{1}$ and $c_{2}$ c-numbers. Tilde operators and non-tilde operators are mutually commutative at equal time:

$$
\left[A_{1}, \tilde{A}_{2}\right]=0 .
$$

Tilde operators and non-tilde operators are related to each other through the thermal state condition for the thermal bra-vacuum $\langle 1|$ :

$$
\langle 1| \tilde{A}=\langle 1| A^{\dagger} .
$$

The dynamics of a system is described by the dissipative Schrödinger equation (1.1) with the initial condition $|0(t=0)\rangle=|0\rangle$. The Hat-Hamiltonian satisfies the tildean condition

$$
(\mathrm{i} \hat{H})^{\sim}=\mathrm{i} \hat{H}
$$

however, it need not be Hermitian. The hat-Hamiltonian has zero eigenvalue for the thermal bra-vacuum:

$$
\langle 1| \hat{H}=0 .
$$

The thermal vacua $\langle 1|$ and $|0\rangle$ are tilde invariant:

$$
\left\langle\left. 1\right|^{\sim}=\langle 1|, \quad| 0\rangle^{\sim}=\mid 0\right\rangle,
$$

and are normalized as

$$
\langle 1 \mid 0\rangle=1
$$

\footnotetext{
${ }^{1}$ For the more information about NETFD, see the original sources, Refs. [5, 6, 7], and the review paper, Ref. [9].

${ }^{2}$ For the formalisms for fermionic systems, see Ref. [10] and references cited therein.

${ }^{3}$ Non-tilde and tilde operators are first introduced in thermo field dynamics [11].
} 
By virtue of (2.7), we have,

$$
|0(t)\rangle^{\sim}=\left(\mathrm{e}^{-\mathrm{i} \hat{H} t}|0\rangle\right)^{\sim}=\mathrm{e}^{-(\mathrm{i} \hat{H})^{\tau} t}|0\rangle^{\sim}=\mathrm{e}^{-\mathrm{i} \hat{H} t}|0\rangle=|0(t)\rangle,
$$

where we have used the formal solution $|0(t)\rangle=\mathrm{e}^{-\mathrm{i} \hat{H} t}|0\rangle$ of (1.1) in the first and fourth equalities. (2.8) implies that

$$
\langle 1 \mid 0(t)\rangle=\left\langle 1\left|\mathrm{e}^{\mathrm{i} \hat{H} t}\right| 0(t)\right\rangle=\langle 1 \mid 0\rangle=1,
$$

i.e., the conservation of probability.

The expectation value of an operator $A$ at time $t$ is given by

$$
\langle A\rangle_{t}:=\langle 1|A| 0(t)\rangle .
$$

Observable operators consist only of non-tilde operators and are Hermitian. We can confirm that the expectation value of an observable operator $A$ is real as

$$
\begin{aligned}
\langle A\rangle_{t}^{*} & =(\langle 1|A| 0(t)\rangle)^{\sim}=\sim\langle 1|\tilde{A}| 0(t)\rangle^{\sim}=\langle 1|\tilde{A}| 0(t)\rangle \\
& =\left\langle 1\left|A^{\dagger}\right| 0(t)\right\rangle=\langle 1|A| 0(t)\rangle=\langle A\rangle_{t} .
\end{aligned}
$$

The expectation value of an operator $A$ can be written as

$$
\langle A\rangle_{t}=\langle 1|A| 0(t)\rangle=\left\langle 1\left|\mathrm{e}^{\mathrm{i} \hat{H} t} A \mathrm{e}^{-\mathrm{i} \hat{H} t}\right| 0\right\rangle=\langle 1|A(t)| 0\rangle .
$$

The new operator $A(t)$ defined by

$$
A(t):=\mathrm{e}^{\mathrm{i} \hat{H} t} A \mathrm{e}^{-\mathrm{i} \hat{H} t},
$$

is the Heisenberg operator satisfying the Heisenberg equation,

$$
\frac{\mathrm{d}}{\mathrm{d} t} A(t)=\mathrm{i}[\hat{H}(t), A(t)],
$$

with $\hat{H}(t)=\mathrm{e}^{\mathrm{i} \hat{H} t} \hat{H} \mathrm{e}^{-\mathrm{i} \hat{H} t}=\hat{H}$.

\section{Annihilation-creation operators of a semi-free field}

Within the density operator method, the quantum master equation describing the dynamics of a damped harmonic oscillator is given by

$$
\frac{\partial}{\partial t} \rho_{S}(t)=-\mathrm{i}\left(H_{0}^{\times}+\mathrm{i} \Pi\right) \rho_{S}(t),
$$

with

$$
\begin{aligned}
H_{0}^{\times} \bullet & :=\left[H_{0}, \bullet\right], \quad H_{0}:=\omega a^{\dagger} a, \\
\Pi \bullet & :=\kappa\left\{\left[a, \bullet a^{\dagger}\right]+\left[a \bullet, a^{\dagger}\right]+2 \bar{n}\left[a,\left[\bullet, a^{\dagger}\right]\right]\right\},
\end{aligned}
$$


where $\rho_{S}(t)$ is the density operator of the system of interest, $a$ is an oscillator operator satisfying the canonical commutation relation,

$$
\left[a, a^{\dagger}\right]=1,
$$

and $\omega, \kappa$ and $\bar{n}$ are positive constants. $H_{0}$ is the free Hamiltonian and $\Pi$ describes the dissipation. See, for example, Ref. [2] for the derivation of the damping operator $\Pi$.

In NETFD, the corresponding dynamics is described by the dissipative Schrödinger equation $[5,6,7,10]$,

$$
\frac{\partial}{\partial t}|W(t)\rangle=-\mathrm{i}\left(\hat{H}_{0}+\mathrm{i} \hat{\Pi}\right)|W(t)\rangle,
$$

with

$$
\begin{aligned}
\hat{H}_{0} & =H_{0}-\tilde{H}_{0}, \quad H_{0}=\omega a^{\dagger} a, \\
\hat{\Pi} & =-\kappa\left[(1+2 \bar{n})\left(a^{\dagger} a+\tilde{a}^{\dagger} \tilde{a}\right)-2(1+\bar{n}) a \tilde{a}-2 \bar{n} a^{\dagger} \tilde{a}^{\dagger}+2 \bar{n}\right],
\end{aligned}
$$

where the operator $\tilde{a}$ satisfies the canonical commutation relation

$$
\left[\tilde{a}, \tilde{a}^{\dagger}\right]=1,
$$

and, for later convenience, we have employed the notation $|W(t)\rangle$ for the thermal ket-vacuum which is subject purely to the dissipation and not to the squeezing. The form (3.7) of $\Pi$ is obtained from (3.3) by the means of "the principle of correspondence" (see Appendix A). It can be also derived axiomatically within the formalism of NETFD [7]. Note that, in general, the operators $a$ and $\tilde{a}$ have subscripts signifying momentum, spin and/or other degrees of freedom as $a_{i}$ and $\tilde{a}_{i}$. However, here and hereafter, we consider operators belonging to a specific index, say, $i$, and, therefore, suppress it for simplicity.

The time-evolution equation for the one-particle distribution function

$$
n(t):=\left\langle 1\left|a^{\dagger} a\right| W(t)\right\rangle,
$$

is given as

$$
\frac{\mathrm{d}}{\mathrm{d} t} n(t)=-2 \kappa[n(t)-\bar{n}],
$$

where we have used (2.6) with $A=a$, i.e.,

$$
\langle 1| \tilde{a}=\langle 1| a^{\dagger},
$$

in the derivation. The equation (3.10) is solved, with the initial condition $n(0)=$ $n$, to give

$$
n(t)=\bar{n}+(n-\bar{n}) \mathrm{e}^{-2 \kappa t} .
$$

We see from (3.12) that $n(t)$ relaxes to $\bar{n}$ for $t \rightarrow \infty . \bar{n}$ is related to the temperature $T$ of the heat bath through the Planck distribution function (in the unit the Boltzmann constant $k_{B}=1$ )

$$
\bar{n}=\frac{1}{\mathrm{e}^{\omega / T}-1} .
$$


The constant $\kappa$ is related to the life time $\tau$, the time in which $|n(t)-\bar{n}|$ decreases by the factor $\mathrm{e}^{-1}$, of the particle as $\kappa=(2 \tau)^{-1}$. When dissipation is present $(\kappa>0)$, the particle has a finite life-time and it can not be considered as free in the limit $t \rightarrow \infty$. The fields $a$ and $\tilde{a}$ are called semi-free when the hatHamiltonian is given in the form that the free part $\hat{H}_{0}$ accompanied with the dissipation part iก̂ of (3.7).

We assume that the initial thermal ket-vacuum $|W(t=0)\rangle=|0\rangle$ is specified by the thermal state condition

$$
\tilde{a}|0\rangle=f a^{\dagger}|0\rangle,
$$

with a real number $f$. The relation between $f$ and the initial number of the particles $n(0)=n$ is obtained by noting that the two expectation values

$$
\langle 1|a \tilde{a}| 0\rangle=f\left\langle 1\left|a a^{\dagger}\right| 0\right\rangle=f(n+1)
$$

and

$$
\langle 1|\tilde{a} a| 0\rangle=\left\langle 1\left|a^{\dagger} a\right| 0\right\rangle=n .
$$

are identical since $[a, \tilde{a}]=0$ from (2.5). Equating the right hand sides of (3.15) and (3.16) yields

$$
n=\frac{f}{1-f} \quad\left(f=\frac{n}{1+n}\right) .
$$

The relation between $n$ and the initial temperature $T_{0}$ of the system is given through the Planck distribution function

$$
n=\frac{1}{\mathrm{e}^{\omega / T_{0}}-1} .
$$

The annihilation-creation operators $\gamma_{t}^{(W)}, \tilde{\gamma}_{t}^{(W)}, \gamma^{\text {क }}$ and $\tilde{\gamma}^{\text {क }}$ associated with the thermal vacua $|W(t)\rangle$ and $\langle 1|$ are introduced by the time-dependent Bogoliubov transformation $[5,6,7,13]$,

$$
\left(\begin{array}{c}
\gamma_{t}^{(W)} \\
\tilde{\gamma}^{\ddagger}
\end{array}\right)=\left(\begin{array}{cc}
1+n(t) & -n(t) \\
-1 & 1
\end{array}\right)\left(\begin{array}{c}
a \\
\tilde{a}^{\dagger}
\end{array}\right),
$$

and its tilde conjugate. For the derivations, see Ref. [14] and references cited therein. The operators $\gamma_{t}^{(W)}, \tilde{\gamma}_{t}^{(W)}, \gamma^{+}$and $\tilde{\gamma}^{+}$annihilate the vacua $|W(t)\rangle$ and $\langle 1|$, i.e.,

$$
\begin{aligned}
\gamma_{t}^{(W)}|W(t)\rangle & =0, & & \tilde{\gamma}_{t}^{(W)}|W(t)\rangle=0, \\
\langle 1| \gamma^{+} & =0, & & \langle 1| \tilde{\gamma}^{+}=0,
\end{aligned}
$$

and satisfy the canonical commutation relations,

$$
\begin{gathered}
{\left[\gamma_{t}^{(W)}, \gamma^{+}\right]=1, \quad\left[\tilde{\gamma}_{t}^{(W)}, \tilde{\gamma}^{+}\right]=1,} \\
{\left[\gamma_{t}^{(W)}, \tilde{\gamma}_{t}^{(W)}\right]=\left[\gamma_{t}^{(W)}, \tilde{\gamma}^{+}\right]=\left[\tilde{\gamma}_{t}^{(W)}, \gamma^{+}\right]=\left[\gamma^{+}, \tilde{\gamma}^{+}\right]=0 .}
\end{gathered}
$$


Reflecting the fact that $\langle 1|$ does not depend on time nor the specific form of the hat-Hamiltonian, $\gamma^{+}$and $\tilde{\gamma}^{+}$do not depend on time nor the form of hatHamiltonian. Thus, $\gamma^{+}$and $\tilde{\gamma}^{+}$do not have the subscript indicating time nor the superscript $(W)$. We will use the notations $\gamma=\gamma_{0}^{(W)}$ and $\tilde{\gamma}=\tilde{\gamma}_{0}^{(W)}$ for the annihilation operators of the initial thermal ket-vacuum $|0\rangle$, i.e.,

$$
\gamma|0\rangle=0, \quad \tilde{\gamma}|0\rangle=0
$$

By using the inverse transformation of (3.19), $\hat{H}_{0}$ of (3.6) and $\hat{\Pi}$ of (3.7) can be rewritten in terms of $\gamma_{t}^{(W)}, \tilde{\gamma}_{t}^{(W)}, \gamma^{+}$and $\tilde{\gamma}^{+}$as

$$
\begin{aligned}
\hat{H}_{0} & =\omega\left(\gamma^{+} \gamma_{t}^{(W)}-\tilde{\gamma}^{+} \tilde{\gamma}_{t}^{(W)}\right) \\
\hat{\Pi} & =-\kappa\left\{\gamma^{+} \gamma_{t}^{(W)}+\tilde{\gamma}^{+} \tilde{\gamma}_{t}^{(W)}+2[n(t)-\bar{n}] \gamma^{+} \tilde{\gamma}^{+}\right\} \\
& =-\kappa\left(\gamma^{+} \gamma_{t}^{(W)}+\tilde{\gamma}^{+} \tilde{\gamma}_{t}^{(W)}\right)+\frac{\mathrm{d} n(t)}{\mathrm{d} t} \gamma^{+} \tilde{\gamma}^{+},
\end{aligned}
$$

where we have used (3.12) in the second equality of (3.26). From (3.5), (3.20), (3.25) and (3.26), we have

$$
\delta|W(t)\rangle=\delta n(t) \gamma^{+} \tilde{\gamma}^{+}|W(t)\rangle
$$

The equation can be integrated to give

$$
|W(t)\rangle=\exp \left\{[n(t)-n] \gamma^{\text {+ }} \tilde{\gamma}^{\text {क }}\right\}|0\rangle .
$$

The equation (3.28) implies that, for a fixed initial vacuum $|0\rangle,|W(t)\rangle$ depends on $t$ only through $n(t)$. From (3.28), we can interpret the dissipative timeevolution of $|W(t)\rangle$ as the condensing of $\gamma^{+} \tilde{\gamma}^{q}$-pairs into the vacuum $|0\rangle$. The quantity $n(t)-n$ is the order parameter which describes the degree of the dissipative time-evolution.

One can see from (3.28) that $|W(t)\rangle$ at any $t$ belongs to the Fock space $\mathcal{H}(t=0)$ built on the initial vacuum $|0\rangle$. Consequently, the Fock spaces $\mathcal{H}(t)$ built on $|W(t)\rangle$ for all $t$ are equivalent. However, the meaning of the equivalence should be taken with a care. Note that (3.28) is originally given in the form of the formal solution of the dissipative Schrödinger equation (3.5), i.e.,

$$
|W(t)\rangle=\mathrm{e}^{-\mathrm{i}\left(\hat{H}_{0}+\mathrm{i} \hat{\Pi}\right) t}|0\rangle,
$$

and that the operator relating $|W(t)\rangle$ and $|0\rangle$ is not unitary as i $\hat{\Pi}$ is not Hermitian. Thus, $|W(t)\rangle$ and $|0\rangle$ are non-unitarily related. In this sense, "equivalent" in the above should be regards as "non-unitarily equivalent". ${ }^{4}$ In virtue of the

\footnotetext{
${ }^{4}$ In Ref. [15], a different physical situation from here is considered. They introduced an Hermitian Hamiltoninan describing the interaction between $A, B$-particles which they regard, respectively, as the system of interest and the reservoir. The Schrödinger equation can be written in the normal ordering with respect to the annihilation-creation operators $A_{t}, A_{t}^{\dagger}, B_{t}, B_{t}^{\dagger}$
} 
equivalence, one can choose $\mathcal{H}\left(t_{0}\right)$ with any $t_{0}$ as the Hilbert space of the physical system or alternatively one can use the description that the Hilbert space $\mathcal{H}(t)$ formally varies with time $t$. Note that such a description is possible since we are considering the kinetic region where the one-particle distribution $n(t)$ varies sufficiently smoothly with respect to time $t$.

\section{Model of the degenerated parametric ampli- fier}

Within the density operator method, the quantum master equation which models the dynamics of a degenerate parametric amplification in a cavity with dissipation is given by

$$
\frac{\partial}{\partial t} \rho_{S}(t)=-\mathrm{i}\left(H_{0}^{\times}+H_{1, t}^{\times}+\mathrm{i} \Pi\right) \rho_{S}(t),
$$

where $H_{0}$ and $\Pi$ are those in (3.2) and (3.3), and

$$
H_{1, t}:=\frac{\mathrm{i} \chi}{2}\left(-a^{2} \mathrm{e}^{2 \mathrm{i} \omega t}+a^{\dagger^{2}} \mathrm{e}^{-2 \mathrm{i} \omega t}\right) \text {. }
$$

$H_{0}$ and i $\Pi$ are, respectively, the free and dissipation parts of Hamiltonian for the photons at cavity mode and $H_{1, t}$ is the part which corresponds to the process of the degenerate parametric amplification, i.e., the cavity mode at frequency $\omega$ is amplified by the pump mode at frequency $2 \omega . \kappa$ is the attenuation rate which is related to the $Q$-value of the cavity through $\kappa=\omega / 2 \pi Q . \quad \bar{n}$ is the number of photons at the temperature $T$ of the heat bath given by (3.13). The real constant $\chi$ is proportional to the amplitude of the pump mode and to the second-order nonlinear susceptibility of the medium in the cavity. It is assumed that the intensity of the pump mode is so large that the influence of the cavity mode on the pump mode can be neglected and that the pump mode can be treated as a classical external mode.

In NETFD, the corresponding dissipative Schrödinger equation reads

$$
\frac{\partial}{\partial t}|0(t)\rangle=-\mathrm{i} \hat{H}_{t}|0(t)\rangle
$$

associated with the vacuum $|0(t)\rangle$ at time $t$ as

$$
(\partial / \partial t)|0(t)\rangle=\sum_{\boldsymbol{k}}\left(c_{\boldsymbol{k}, 1}(t)+c_{\boldsymbol{k}, 2}(t) A_{\boldsymbol{k}, t}^{\dagger} B_{\boldsymbol{k}, t}^{\dagger}\right)|0(t)\rangle,
$$

where we have restored the subscripts indicating the momentum $\boldsymbol{k}$ and $c_{\boldsymbol{k}, i}(t)(i=1,2)$ are c-number functions. The term $\sum_{\boldsymbol{k}} c_{\boldsymbol{k}, 1}(t) \sim\left[V /(2 \pi)^{3}\right] \int \mathrm{d}^{3} \boldsymbol{k} c_{\boldsymbol{k}, 1}(t)$ diverges as $O(V)$ in the limit of $V \rightarrow \infty$ where $V$ is the volume of the system. It implies the inequivalence of the vacua at different times. Thus, within their formalism, $\mathcal{H}(t)$ at different times $t$ are "unitarily inequivalent". In NETFD, due to the specific form of the non-Hermitian i $\hat{\Pi}$ in (3.26), the term corresponding to $c_{\boldsymbol{k}, 1}(t)$ vanishes [read $\gamma^{+}, \tilde{\gamma}^{+}$as $A_{t}^{\dagger}, B_{t}^{\dagger}$, respectively, in (3.27)]. Consequently, the vacua at different times $t$ are "non-unitarily related", i.e., $\mathcal{H}(t)$ at different times $t$ are "non-unitarily equivalent". Thus, the dissipative time evolution of the vacuum is appropriately treated within NETFD. A detailed analysis on the topic will be reported elsewhere in a near future. 
with

$$
\begin{aligned}
\hat{H}_{t} & :=\hat{H}_{0}+\hat{H}_{1, t}+\mathrm{i} \hat{\Pi}, \\
\hat{H}_{1, t} & :=H_{1, t}-\tilde{H}_{1, t},
\end{aligned}
$$

and $\hat{H}_{0}$ and $\hat{\Pi}$ being those of (3.6) and (3.7). We assume that the system is at the thermal equilibrium state with temperature $T_{0}$ at the initial time $t=0$. Hence, (3.14) with (3.17) and (3.18) applies for the initial thermal ket-vacuum $|0(t=0)\rangle=|0\rangle$.

The thermal ket-vacuum $|0(t)\rangle_{\mathrm{I}}$ in the interaction representation is introduced by

$$
|0(t)\rangle_{\mathrm{I}}:=\mathrm{e}^{\mathrm{i} \hat{H}_{0} t}|0(t)\rangle .
$$

From (4.3) and (4.6), we obtain the time evolution equation of $|0(t)\rangle_{\mathrm{I}}$ as

$$
\frac{\partial}{\partial t}|0(t)\rangle_{\mathrm{I}}=-\mathrm{i}\left[\hat{H}_{1, \mathrm{I}}(t)+\mathrm{i} \hat{\Pi}_{\mathrm{I}}(t)\right]|0(t)\rangle_{\mathrm{I}},
$$

where

$$
\begin{aligned}
\hat{H}_{1, \mathrm{I}}(t) & :=H_{1, \mathrm{I}}(t)-\tilde{H}_{1, \mathrm{I}}(t), \\
H_{1, \mathrm{I}}(t) & :=\mathrm{e}^{\mathrm{i} \hat{H}_{0} t} H_{1, t} \mathrm{e}^{-\mathrm{i} \hat{H}_{0} t}=\frac{\mathrm{i} \chi}{2}\left(-a^{2}+a^{\dagger^{2}}\right)=: H_{1}, \\
\hat{\Pi}_{\mathrm{I}}(t) & :=\mathrm{e}^{\mathrm{i} \hat{H}_{0} t} \hat{\Pi} \mathrm{e}^{-\mathrm{i} \hat{H}_{0} t}=\hat{\Pi} .
\end{aligned}
$$

By substituting (4.8)-(4.10) into (4.7), we have

$$
\frac{\partial}{\partial t}|0(t)\rangle_{\mathrm{I}}=-\mathrm{i} \hat{H}|0(t)\rangle_{\mathrm{I}},
$$

with

$$
\begin{aligned}
\hat{H} & :=\hat{H}_{1}+\mathrm{i} \hat{\Pi}, \\
\hat{H}_{1} & :=H_{1}-\tilde{H}_{1} .
\end{aligned}
$$

\section{Derivation of annihilation-creation operators for dissipative squeezed vacuum}

In the following of this paper, we drop the subscript I in (4.11) and formally deal with a system that evolves according to the dissipative Schrödinger equation

$$
\frac{\partial}{\partial t}|0(t)\rangle=-\mathrm{i} \hat{H}|0(t)\rangle,
$$

with the time-independent hat-Hamiltonian (4.12).

Let $|0(t)\rangle_{\chi=0}$ be the dissipative thermal ket-vacuum without squeezing which evolves in time as

$$
\frac{\partial}{\partial t}|0(t)\rangle_{\chi=0}=\hat{\Pi}|0(t)\rangle_{\chi=0} .
$$


The vacuum $|0(t)\rangle_{\chi=0}$ is related to the vacuum of the semi-free field $|W(t)\rangle$ by

$$
|0(t)\rangle_{\chi=0}=\mathrm{e}^{\mathrm{i} \hat{H}_{0} t}|W(t)\rangle
$$

From (3.20), (3.25) and (5.3), we find that $|0(t)\rangle_{\chi=0}$ is identical to $|W(t)\rangle$, i.e.,

$$
|0(t)\rangle_{\chi=0}=|W(t)\rangle .
$$

Our aim in this section is to derive an expression for the annihilation-creation operators of $|0(t)\rangle$ in (5.1) with reference to those of $|0(t)\rangle_{\chi=0}(=|W(t)\rangle)$, i.e., $\gamma_{t}^{(W)}, \tilde{\gamma}_{t}^{(W)}, \gamma^{\text {q }}$ and $\tilde{\gamma}^{\text {q. }}$.

Let us introduce the "Heisenberg operators" associated with $\gamma_{t}^{(W)}, \tilde{\gamma}_{t}^{(W)}, \gamma^{\text {+ }}$ and $\tilde{\gamma}^{\text {+ }}$ by

$$
\gamma^{(W)}(t)=\mathrm{e}^{\mathrm{i} \hat{H} t} \gamma_{t}^{(W)} \mathrm{e}^{-\mathrm{i} \hat{H} t}, \quad \gamma^{q}(t)=\mathrm{e}^{\mathrm{i} \hat{H} t} \gamma^{q} \mathrm{e}^{-\mathrm{i} \hat{H} t},
$$

and their tilde conjugates. By using the inverse transformation of (3.19) and (3.10), $\hat{\Pi}$ of (3.7) and $\hat{H}_{1}$ of (4.13) can be rewritten in terms of $\gamma_{t}^{(W)}, \tilde{\gamma}_{t}^{(W)}, \gamma^{q}$ and $\tilde{\gamma}^{+}$as

$$
\begin{aligned}
\hat{\Pi} & =-\kappa\left(\gamma^{q} \gamma_{t}^{(W)}+\tilde{\gamma}^{q} \tilde{\gamma}_{t}^{(W)}\right)+\frac{\mathrm{d} n(t)}{\mathrm{d} t} \gamma^{q} \tilde{\gamma}^{q}, \\
\hat{H}_{1} & =\mathrm{i} \chi\left\{\gamma^{q} \tilde{\gamma}_{t}^{(W)}+\tilde{\gamma}^{q} \gamma_{t}^{(W)}+\left[n(t)+\frac{1}{2}\right]\left(\gamma^{q^{2}}+\tilde{\gamma}^{q^{2}}\right)\right\} .
\end{aligned}
$$

The time-evolution equations for $\gamma^{(W)}(t), \tilde{\gamma}^{(W)}(t), \gamma^{q}(t)$ and $\tilde{\gamma}^{\mathcal{9}}(t)$ are given by

$$
\begin{aligned}
\frac{\mathrm{d}}{\mathrm{d} t}\left(\begin{array}{c}
\gamma^{(W)}(t) \\
\tilde{\gamma}^{(W)}(t)
\end{array}\right) & =\mathrm{i}\left[\hat{H}(t),\left(\begin{array}{c}
\gamma^{(W)}(t) \\
\tilde{\gamma}^{(W)}(t)
\end{array}\right)\right]+\mathrm{e}^{\mathrm{i} \hat{H} t} \frac{\mathrm{d}}{\mathrm{d} t}\left(\begin{array}{c}
\gamma^{(W)}(t) \\
\tilde{\gamma}^{(W)}(t)
\end{array}\right) \mathrm{e}^{-\mathrm{i} \hat{H} t} \\
& =-K\left(\begin{array}{c}
\gamma^{(W)}(t) \\
\tilde{\gamma}^{(W)}(t)
\end{array}\right)+\chi[2 n(t)+1]\left(\begin{array}{c}
\gamma^{+}(t) \\
\tilde{\gamma}^{+}(t)
\end{array}\right), \\
\frac{\mathrm{d}}{\mathrm{d} t}\left(\gamma^{+}(t), \tilde{\gamma}^{+}(t)\right) & =\mathrm{i}\left[\hat{H}(t),\left(\gamma^{+}(t), \tilde{\gamma}^{+}(t)\right)\right] \\
& =\left(\gamma^{+}(t), \tilde{\gamma}^{+}(t)\right) K,
\end{aligned}
$$

where

$$
K:=\left(\begin{array}{cc}
\kappa & -\chi \\
-\chi & \kappa
\end{array}\right)
$$

The equations (5.8) and (5.9) can be solved with the initial conditions $\gamma^{(W)}(0)=$ $\gamma, \tilde{\gamma}^{(W)}(0)=\tilde{\gamma}, \gamma^{q}(0)=\gamma^{q}$ and $\tilde{\gamma}^{q}(0)=\tilde{\gamma}^{q}$ as

$$
\begin{aligned}
\left(\begin{array}{c}
\gamma^{(W)}(t) \\
\tilde{\gamma}^{(W)}(t)
\end{array}\right) & =\mathrm{e}^{-K t}\left(\begin{array}{c}
\gamma \\
\tilde{\gamma}
\end{array}\right)+\int_{0}^{t} \mathrm{~d} t^{\prime} \chi\left[2 n\left(t^{\prime}\right)+1\right] \mathrm{e}^{K\left(2 t^{\prime}-t\right)}\left(\begin{array}{l}
\gamma^{+} \\
\tilde{\gamma}^{+}
\end{array}\right), \\
\left(\gamma^{+}(t), \tilde{\gamma}^{+}(t)\right) & =\left(\gamma^{q}, \tilde{\gamma}^{\text {q }}\right) \mathrm{e}^{K t} .
\end{aligned}
$$


It can be shown that $\gamma^{+}(t)$ and $\tilde{\gamma}^{+}(t)$ annihilate the thermal bra-vacuum $\langle 1|$ as follows:

$$
\langle 1|\left(\gamma^{q}(t), \tilde{\gamma}^{q}(t)\right)=\langle 1| \mathrm{e}^{\mathrm{i} \hat{H} t}\left(\gamma^{q}, \tilde{\gamma}^{q}\right) \mathrm{e}^{-\mathrm{i} \hat{H} t}=\langle 1|\left(\gamma^{q}, \tilde{\gamma}^{q}\right) \mathrm{e}^{-\mathrm{i} \hat{H} t}=(0,0),
$$

where we have used (2.8) for the second equality and (3.21) for the last equality. Since $\gamma$ and $\tilde{\gamma}$ are the annihilation operators for $|0\rangle$, the operators $\gamma(t)$ and $\tilde{\gamma}(t)$ which annihilate $|0\rangle$, i.e,

$$
\left(\begin{array}{l}
\gamma(t) \\
\tilde{\gamma}(t)
\end{array}\right)|0\rangle=\left(\begin{array}{l}
0 \\
0
\end{array}\right)
$$

can be introduced by

$$
\begin{aligned}
\left(\begin{array}{l}
\gamma(t) \\
\tilde{\gamma}(t)
\end{array}\right) & =C(t)\left(\begin{array}{l}
\gamma \\
\tilde{\gamma}
\end{array}\right) \\
& =C(t)\left[\mathrm{e}^{K t}\left(\begin{array}{c}
\gamma^{(W)}(t) \\
\tilde{\gamma}^{(W)}(t)
\end{array}\right)-\int_{0}^{t} \mathrm{~d} t^{\prime} \chi\left[2 n\left(t^{\prime}\right)+1\right] \mathrm{e}^{K\left(2 t^{\prime}-t\right)}\left(\begin{array}{c}
\gamma^{q}(t) \\
\tilde{\gamma}^{+}(t)
\end{array}\right)\right],
\end{aligned}
$$

where $C(t)$ is a matrix and we have used (5.11) and (5.12) for the second equality. In order that the canonical commutation relation is satisfied for $\gamma(t), \tilde{\gamma}(t), \gamma^{q}(t)$ and $\tilde{\gamma}^{+}(t)$, we have

$$
\left(\begin{array}{ll}
1 & 0 \\
0 & 1
\end{array}\right)=\left[\left(\begin{array}{c}
\gamma(t) \\
\tilde{\gamma}(t)
\end{array}\right),\left(\gamma^{+}(t), \tilde{\gamma}^{+}(t)\right)\right]=C(t)\left[\left(\begin{array}{c}
\gamma \\
\tilde{\gamma}
\end{array}\right),\left(\gamma^{+}, \tilde{\gamma}^{+}\right)\right] \mathrm{e}^{K t}=C(t) \mathrm{e}^{K t}
$$

Thus,

$$
C(t)=\mathrm{e}^{-K t} .
$$

The Schrödinger operators $\gamma_{t}, \tilde{\gamma}_{t}, \gamma_{t}^{+}$and $\tilde{\gamma}_{t}^{\text {}}$ associated, respectively, with $\gamma(t), \tilde{\gamma}(t), \gamma^{\mathcal{\rho}}(t)$ and $\tilde{\gamma}^{\mathcal{P}}(t)$ can be introduced by the relations

$$
\gamma(t)=\mathrm{e}^{\mathrm{i} \hat{H} t} \gamma_{t} \mathrm{e}^{-\mathrm{i} \hat{H} t}, \quad \gamma^{\uparrow}(t)=\mathrm{e}^{\mathrm{i} \hat{H} t} \gamma_{t}^{\uparrow} \mathrm{e}^{-\mathrm{i} \hat{H} t}
$$

and their tilde conjugates. Using (5.5), (5.12), (5.15), (5.17) and (5.18), we arrive at

$$
\begin{aligned}
\left(\begin{array}{c}
\gamma_{t} \\
\tilde{\gamma}_{t}
\end{array}\right) & =\left(\begin{array}{c}
\gamma_{t}^{(W)} \\
\tilde{\gamma}_{t}^{(W)}
\end{array}\right)-\int_{0}^{t} \mathrm{~d} t^{\prime} \chi\left[2 n\left(t^{\prime}\right)+1\right] \mathrm{e}^{2 K\left(t^{\prime}-t\right)}\left(\begin{array}{c}
\gamma^{\text {q }} \\
\tilde{\gamma}^{+}
\end{array}\right), \\
\left(\gamma_{t}^{+}, \tilde{\gamma}_{t}^{+}\right) & =\left(\gamma^{q}, \tilde{\gamma}^{\text {q }}\right) .
\end{aligned}
$$

In the following, we will drop the subscripts $t$ in $\gamma_{t}^{q}$ and $\tilde{\gamma}_{t}^{q}$ since they are time-independent and identical to $\gamma^{q}$ and $\tilde{\gamma}^{q}$, respectively. We have

$$
\left(\begin{array}{c}
\gamma_{t} \\
\tilde{\gamma}_{t}
\end{array}\right)|0(t)\rangle=\mathrm{e}^{-\mathrm{i} \hat{H} t}\left(\begin{array}{c}
\gamma(t) \\
\tilde{\gamma}(t)
\end{array}\right) \mathrm{e}^{\mathrm{i} \hat{H} t} \mathrm{e}^{-\mathrm{i} \hat{H} t}|0\rangle=\mathrm{e}^{-\mathrm{i} \hat{H} t}\left(\begin{array}{c}
\gamma(t) \\
\tilde{\gamma}(t)
\end{array}\right)|0\rangle=\left(\begin{array}{c}
0 \\
0
\end{array}\right),
$$


where we have used (5.18) and the formal solution $|0(t)\rangle=\mathrm{e}^{-\mathrm{i} \hat{H} t}|0\rangle$ of (5.1) in the first equality, and (5.14) in the last equality. We have

$$
\begin{aligned}
{\left[\left(\begin{array}{c}
\gamma_{t} \\
\tilde{\gamma}_{t}
\end{array}\right),\left(\gamma^{+}, \tilde{\gamma}^{+}\right)\right] } & =\left[\mathrm{e}^{-\mathrm{i} \hat{H} t}\left(\begin{array}{c}
\gamma(t) \\
\tilde{\gamma}(t)
\end{array}\right) \mathrm{e}^{\mathrm{i} \hat{H} t}, \mathrm{e}^{-\mathrm{i} \hat{H} t}\left(\gamma^{+}(t), \tilde{\gamma}^{+}(t)\right) \mathrm{e}^{\mathrm{i} \hat{H} t}\right] \\
& =\mathrm{e}^{-\mathrm{i} \hat{H} t}\left[\left(\begin{array}{c}
\gamma(t) \\
\tilde{\gamma}(t)
\end{array}\right),\left(\gamma^{+}(t), \tilde{\gamma}^{+}(t)\right)\right] \mathrm{e}^{\mathrm{i} \hat{H} t} \\
& =\left(\begin{array}{cc}
1 & 0 \\
0 & 1
\end{array}\right)
\end{aligned}
$$

where we have used (5.18) in the first equality, and the first equality of (5.16) in the last equality. Thus, we obtained the expressions (5.19) and (5.20) of the annihilation-creation operators $\gamma_{t}, \tilde{\gamma}_{t}, \gamma^{+}$and $\tilde{\gamma}^{+}$that annihilate vacua $|0(t)\rangle$ and $\langle 1|$ as (5.21) and (3.21), and that satisfy the canonical commutation relation (5.22).

Let us introduce a new set of operators $\xi_{t}, \eta_{t}, \xi^{+}$and $\eta^{+}$by

$$
\left(\begin{array}{c}
\xi_{t} \\
\eta_{t}
\end{array}\right):=\Lambda\left(\begin{array}{c}
\gamma_{t} \\
\tilde{\gamma}_{t}
\end{array}\right), \quad\left(\xi^{+}, \eta^{+}\right):=\left(\gamma^{+}, \tilde{\gamma}^{+}\right) \Lambda^{-1},
$$

where

$$
\Lambda:=\frac{1}{\sqrt{2}}\left(\begin{array}{cc}
1 & 1 \\
-\mathrm{i} & \mathrm{i}
\end{array}\right)
$$

is the matrix which diagonalizes $K$ as

$$
K=\Lambda^{-1}\left(\begin{array}{cc}
\kappa-\chi & 0 \\
0 & \kappa+\chi
\end{array}\right) \Lambda
$$

It is evident from $(3.21),(5.21),(5.22)$ and $(5.23)$ that $\xi_{t}, \eta_{t}, \xi^{+}$and $\eta^{+}$annihilate the vacua $|0(t)\rangle$ and $\langle 1|$, i.e.,

$$
\left(\begin{array}{c}
\xi_{t} \\
\eta_{t}
\end{array}\right)|0(t)\rangle=\left(\begin{array}{l}
0 \\
0
\end{array}\right), \quad\langle 1|\left(\xi^{\text {o }}, \eta^{\text {o }}\right)=(0,0),
$$

and satisfy the canonical commutation relation

$$
\left[\left(\begin{array}{l}
\xi_{t} \\
\eta_{t}
\end{array}\right),\left(\xi^{+}, \eta^{+}\right)\right]=\left(\begin{array}{ll}
1 & 0 \\
0 & 1
\end{array}\right) .
$$

Furthermore, (5.23) and (5.24) imply that the operators are invariant under the tilde conjugation,

$$
\tilde{\xi}_{t}=\xi_{t}, \quad \tilde{\eta}_{t}=\eta_{t}, \quad \tilde{\xi}^{\text {+ }}=\xi^{+}, \quad \tilde{\eta}^{\text {+ }}=\eta^{\text {+ }} .
$$

From (5.19),(5.23) and (5.25), we obtain

$$
\left(\begin{array}{c}
\xi_{t} \\
\eta_{t}
\end{array}\right)=\Lambda\left(\begin{array}{l}
\gamma_{t}^{(W)} \\
\tilde{\gamma}_{t}^{(W)}
\end{array}\right)-\left(\begin{array}{cc}
s_{+}(t) & 0 \\
0 & -s_{-}(t)
\end{array}\right) \Lambda\left(\begin{array}{l}
\gamma^{\circ} \\
\tilde{\gamma}^{q}
\end{array}\right)
$$


with

$$
\begin{aligned}
s_{ \pm}(t) & := \pm \chi \int_{0}^{t} \mathrm{~d} t^{\prime}\left[2 n\left(t^{\prime}\right)+1\right] \mathrm{e}^{2(-\kappa \pm \chi)\left(t-t^{\prime}\right)} \\
& =n-n(t)+\frac{ \pm \chi\left(\bar{n}+\frac{1}{2}\right)-\kappa(n-\bar{n})}{\kappa \mp \chi}\left(1-\mathrm{e}^{-2(\kappa \pm \chi) t}\right),
\end{aligned}
$$

where (3.12) is used for the second equality of (5.30).

\section{Quadrature-phase amplitudes}

The quadrature-phase amplitudes $q$ and $p$, defined by

$$
\left(\begin{array}{c}
q \\
p
\end{array}\right):=\Lambda\left(\begin{array}{c}
a \\
a^{\dagger}
\end{array}\right)
$$

satisfy the canonical commutation relation,

$$
[q, p]=\mathrm{i} .
$$

Let $x(t)$ and $y(t)$ be the vacuum expectation values of $q$ and $p$ at time $t$, and $\Delta x^{2}(t)$ and $\Delta y^{2}(t)$ be the variances,

$$
\begin{gathered}
x(t):=\langle 1|q| 0(t)\rangle, \quad y(t):=\langle 1|p| 0(t)\rangle, \\
\Delta x^{2}(t):=\left\langle 1\left|[q-x(t)]^{2}\right| 0(t)\right\rangle, \quad \Delta y^{2}(t):=\left\langle 1\left|[p-y(t)]^{2}\right| 0(t)\right\rangle .
\end{gathered}
$$

By using the inverse transformation of (3.19), the second equality of (5.23), (5.29) and (6.1), $q$ and $p$ can be written in terms of $\xi_{t}, \eta_{t}, \xi^{\text {+ }}$ and $\eta^{+}$as

$$
\left(\begin{array}{l}
q \\
p
\end{array}\right)=\left(\begin{array}{c}
\xi_{t} \\
\eta_{t}
\end{array}\right)+\left(\begin{array}{cc}
n(t)+\frac{1}{2}+s_{+}(t) & -\frac{i}{2} \\
\frac{i}{2} & n(t)+\frac{1}{2}+s_{-}(t)
\end{array}\right)\left(\begin{array}{c}
\xi^{+} \\
\eta^{+}
\end{array}\right) .
$$

Hence, we have

$$
\begin{array}{cl}
x(t)=0, & y(t)=0, \\
\Delta x^{2}(t)=n(t)+\frac{1}{2}+s_{+}(t), & \Delta y^{2}(t)=n(t)+\frac{1}{2}+s_{-}(t) .
\end{array}
$$

The results (6.6) and (6.7) are consistent with those obtained based on the density operator method in Ref. [4].

The time-evolution equations for $\Delta x^{2}(t)$ and $\Delta y^{2}(t)$ are given by

$$
\begin{gathered}
\frac{\mathrm{d}}{\mathrm{d} t}\left(\Delta x^{2}(t)\right)=2\left[\chi\left(n+\frac{1}{2}\right)-\kappa(n-\bar{n})\right] \mathrm{e}^{-2(\kappa-\chi) t}, \\
\frac{\mathrm{d}}{\mathrm{d} t}\left(\Delta y^{2}(t)\right)=2\left[-\chi\left(n+\frac{1}{2}\right)-\kappa(n-\bar{n})\right] \mathrm{e}^{-2(\kappa+\chi) t},
\end{gathered}
$$


where (3.10),(5.30) and (6.7) are used in the derivation.

From the inverse transformation of (6.1), (6.4), (6.6) and (6.7), the number of photons of cavity mode $N(t)$ is given by

$$
\begin{aligned}
N(t) & :=\left\langle 1\left|a^{\dagger} a\right| 0(t)\right\rangle \\
& =\frac{1}{2}\left[\Delta x^{2}(t)+\Delta y^{2}(t)-1\right] \\
& =n(t)+\frac{s_{+}(t)+s_{-}(t)}{2} .
\end{aligned}
$$

The difference

$$
\begin{aligned}
\Delta(t) & :=\Delta x^{2}(t)-\Delta y^{2}(t) \\
& =s_{+}(t)-s_{-}(t),
\end{aligned}
$$

can be considered as a measure of squeezing.

\section{Structure of the vacuum $|0(t)\rangle$}

By using the inverse transformations of the second equality of (5.23) and (5.29), $\hat{\Pi}$ of (5.6) and $\hat{H}_{1}$ of (5.7) can be rewritten in terms of $\xi_{t}, \eta_{t}, \xi^{+}$and $\eta^{+}$as

$$
\begin{gathered}
\hat{\Pi}=-\kappa\left\{\xi^{+} \xi_{t}+\eta^{+} \eta_{t}+\left[s_{+}(t)+(n(t)-\bar{n})\right] \xi^{q^{2}}+\left[s_{-}(t)+(n(t)-\bar{n})\right] \eta^{q^{2}}\right\}, \\
\hat{H}_{1}=\mathrm{i} \chi\left\{\xi^{+} \xi_{t}-\eta^{+} \eta_{t}+\left[s_{+}(t)+n(t)+\frac{1}{2}\right] \xi^{\xi^{2}}-\left[s_{-}(t)+n(t)+\frac{1}{2}\right] \eta^{q^{2}}\right\} .
\end{gathered}
$$

Substituting (7.1) and (7.2) with (5.30) into (5.1) and noting (6.8) and (6.9), we obtain

$$
\delta|0(t)\rangle=\left\{\frac{1}{2} \delta\left[\Delta x^{2}(t)\right]{\xi^{q^{2}}}^{2}+\frac{1}{2} \delta\left[\Delta y^{2}(t)\right]{\eta^{q^{2}}}^{2}|0(t)\rangle .\right.
$$

The equation can be integrated with the initial condition $|0(t=0)\rangle=|0\rangle$ as

$$
|0(t)\rangle=\exp \left[\frac{\Delta x^{2}(t)-\Delta x^{2}(0)}{2} \xi^{\rho^{2}}+\frac{\Delta y^{2}(t)-\Delta y^{2}(0)}{2} \eta^{q^{2}}\right]|0\rangle .
$$

The expression (7.4) implies that the dissipative squeezing process can be interpreted as the condensing of pairs $\xi^{+} \xi^{q}$ and $\eta^{+} \eta^{q}$ into the vacuum $|0\rangle$. The order parameters corresponding to pairs $\xi^{+} \xi^{+}$and $\eta^{+} \eta^{+}$are the changes of variances, respectively, $\Delta x^{2}(t)-\Delta x^{2}(0)$ and $\Delta y^{2}(t)-\Delta y^{2}(0)$.

From (5.23), (6.10), (6.11) and (7.4), an alternative expression of $|0(t)\rangle$ is obtained as

$$
|0(t)\rangle=\exp \left\{[N(t)-n] \gamma^{q} \tilde{\gamma}^{q}+\frac{\Delta(t)}{4}\left({\gamma^{q^{2}}}^{2}+\tilde{\gamma}^{q^{2}}\right)\right\}|0\rangle,
$$


where we have used $N(0)=n$ and $\Delta(0)=0$ by noting $s_{ \pm}(0)=0$ [see (5.30)]. Note that $\hat{\Pi}$ is invariant under the phase transformation $\left(a, a^{\dagger}, \tilde{a}, \tilde{a}^{\dagger}\right) \rightarrow$ $\left(\mathrm{e}^{\mathrm{i} \phi} a, \mathrm{e}^{-\mathrm{i} \phi} a^{\dagger}, \mathrm{e}^{-\mathrm{i} \phi} \tilde{a}, \mathrm{e}^{\mathrm{i} \phi} \tilde{a}^{\dagger}\right)$, while $\hat{H}_{1}$ is not. Thus, $\hat{H}_{1}$ breaks the phase symmetry of the system. The quantity $\Delta(t)$ is the order parameter which corresponds to breakdown of the phase symmetry. The expression (7.5) allows us to interpret the breakdown of the phase symmetry as the condensation of the combination of pairs $\left(\gamma^{+} \gamma^{+}+\tilde{\gamma}^{+} \tilde{\gamma}^{+}\right)$into the vacuum $|0\rangle$.

The equation (7.5) implies that, for a fixed initial vacuum $|0\rangle$, the dissipative squeezed vacuum $|0(t)\rangle$ is determined by $N(t)$ and $\Delta(t)$ and it depends on time $t$ only through $N(t)$ and $\Delta(t)$. Let us introduce the notation,

$$
|0(N, \Delta)\rangle:=\exp \left\{[N-n] \gamma^{q} \tilde{\gamma}^{q}+\frac{\Delta}{4}\left(\gamma^{q^{2}}+\tilde{\gamma}^{q^{2}}\right)\right\}|0\rangle .
$$

Then, the vacuum $|0(N, \Delta)\rangle$ can be expressed in relation to a reference vacuum $\left|0\left(N_{R}, \Delta_{R}\right)\right\rangle$ as

$$
|0(N, \Delta)\rangle=\exp \left\{\left[N-N_{R}\right] \gamma^{q} \tilde{\gamma}^{q}+\frac{\Delta-\Delta_{R}}{4}\left(\gamma^{q^{2}}+\tilde{\gamma}^{q^{2}}\right)\right\}\left|0\left(N_{R}, \Delta_{R}\right)\right\rangle .
$$

If we choose the purely dissipative vacuum $|W(t)\rangle=|0(n(t), 0)\rangle$ [see (3.28)] as the reference vacuum, i.e., $N_{R}=n(t)$ and $\Delta_{R}=0$, we obtain the expression of the dissipative squeezed vacuum $|0(t)\rangle$ as

$$
|0(t)\rangle=\exp \left\{[N(t)-n(t)] \gamma^{q} \tilde{\gamma}^{+}+\frac{\Delta(t)}{4}\left(\gamma^{q^{2}}+\tilde{\gamma}^{q^{2}}\right)\right\}|W(t)\rangle .
$$

The expression (7.8) tells us that, besides the effect of pure dissipation, there is an extra condensation of $\gamma^{+} \tilde{\gamma}^{+}$-pairs due to the dissipative squeezing process.

The squeezed ket-vacuum without dissipation is given by

$$
|S(t)\rangle:=|0(t)\rangle_{\kappa=0}=\left|0\left(N_{0}(t), \Delta_{0}(t)\right)\right\rangle,
$$

where

$$
\begin{aligned}
& N_{0}(t):=\left.N(t)\right|_{\kappa=0}=\left(n+\frac{1}{2}\right) \cosh (2 \chi t)-\frac{1}{2}, \\
& \Delta_{0}(t):=\left.\Delta(t)\right|_{\kappa=0}=\left(n+\frac{1}{2}\right) 2 \sinh (2 \chi t) .
\end{aligned}
$$

If we choose $|S(t)\rangle$ as the reference vacuum, i.e., $N_{R}=N_{0}(t)$ and $\Delta_{R}=\Delta_{0}(t)$,we obtain the expression of $|0(t)\rangle$ as

$$
|0(t)\rangle=\exp \left\{\left[N(t)-N_{0}(t)\right] \gamma^{q} \tilde{\gamma}^{q}+\frac{\Delta(t)-\Delta_{0}(t)}{4}\left(\gamma^{q^{2}}+\tilde{\gamma}^{q^{2}}\right)\right\}|S(t)\rangle .
$$

The effect of the dissipation can be regarded as the condensation of $\gamma^{+} \tilde{\gamma}^{+}$-pairs and that of the combination of pairs $\left(\gamma^{q} \gamma^{q}+\tilde{\gamma}^{+} \tilde{\gamma}^{q}\right)$. 

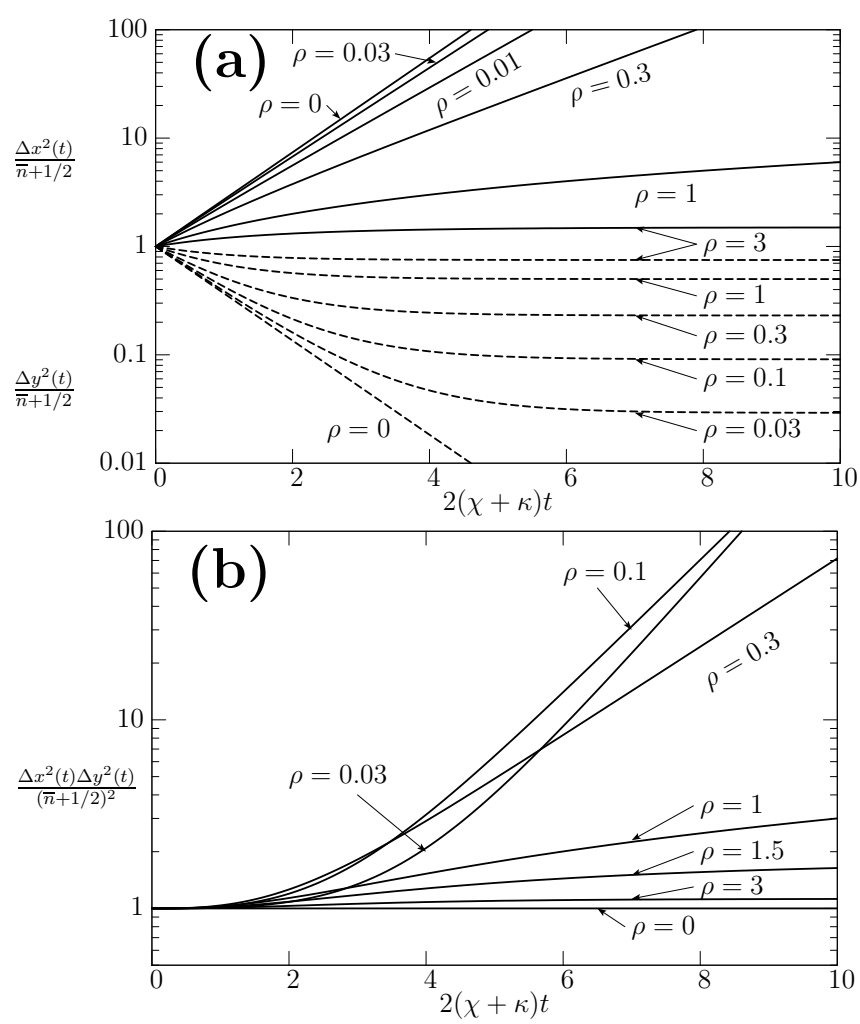

Figure 1: (a) Time evolutions of the variances of the quadrature amplitudes $\Delta x^{2}(t)$ (solid lines) and $\Delta y^{2}(t)$ (dashed lines) normalized by $\bar{n}+1 / 2$ for the ratios $\rho(=\kappa / \chi)=0,0.03,0.1,0.3,1$ and 3 . The time is normalized in the unit of the time scale $[2(\chi+\kappa)]^{-1}$. (b) Time evolution of the square of the uncertainty relation $\Delta x^{2}(t) \Delta y^{2}(t)$ normalized by $(\bar{n}+1 / 2)^{2}$ for $\rho=0,0.03,0.1,0.3,1,1.5$ and 3 .

\section{Effect of dissipation on squeezing and uncer- tainty relations}

Let us consider the implication of (6.7) with (5.30). For simplicity, let us consider the case that $n=\bar{n}$. This corresponds to the case that the cavity is initially at the thermal equilibrium state with temperature $T=\omega /[\ln (\bar{n}+1 / \bar{n})]$ and that the system is coupled to a heat bath with the same temperature $T$ throughout the squeezing process. Let $\chi>0$, then we have $\Delta y^{2}(t)-\Delta y^{2}(0)=s_{-}(t) \leq 0$, thus the vacuum is squeezed in $y$-direction. 
From (6.7) and (5.30), we can write $\Delta x^{2}(t)$ and $\Delta y^{2}(t)$ as

$$
\begin{aligned}
& \Delta x^{2}(t)=\left\{\begin{array}{ll}
\left(\bar{n}+\frac{1}{2}\right) \frac{\kappa}{\kappa-\chi}\left(1-\frac{\chi}{\kappa} \mathrm{e}^{-2(\kappa-\chi) t}\right) & (\kappa \neq \chi) \\
\left(\bar{n}+\frac{1}{2}\right)(1+2 \kappa t) & (\kappa=\chi)
\end{array},\right. \\
& \Delta y^{2}(t)=\left(\bar{n}+\frac{1}{2}\right) \frac{\kappa}{\kappa+\chi}\left(1+\frac{\chi}{\kappa} \mathrm{e}^{-2(\kappa+\chi) t}\right) .
\end{aligned}
$$

The variance $\Delta x^{2}(t)$ saturates in the limit $t \rightarrow \infty$ to

$$
\Delta x_{\max }^{2}:=\lim _{t \rightarrow \infty} \Delta x^{2}(t)=\left(\bar{n}+\frac{1}{2}\right) \frac{\kappa}{\kappa-\chi},
$$

for $\kappa>\chi$, and grows exponentially for $\kappa<\chi$, with the characteristic time $\tau_{x}=1 / 2|\kappa-\chi|$. For the marginal case $\kappa=\chi$, we have the scaling $\Delta x(t) \sim t$. On the other hand, $\Delta y^{2}(t)$ converges to

$$
\Delta y_{\min }^{2}:=\lim _{t \rightarrow \infty} \Delta y^{2}(t)=\left(\bar{n}+\frac{1}{2}\right) \frac{\kappa}{\kappa+\chi},
$$

with the characteristic time $\tau_{y}=1 / 2(\kappa+\chi)$. See Fig. 1(a) for the behaviors of $\Delta x^{2}(t)$ and $\Delta y^{2}(t)$ for the typical values of the ratio $\rho:=\kappa / \chi$.

From (8.1) and (8.2), the square of the uncertainty relation $\Delta x^{2}(t) \Delta y^{2}(t)$ is given by

$$
\Delta x^{2}(t) \Delta y^{2}(t)=\left\{\begin{array}{ll}
\left(\bar{n}+\frac{1}{2}\right)^{2} \frac{\kappa^{2}}{\kappa^{2}-\chi^{2}}\left(1-\mathrm{e}^{-2(\kappa-\chi) t}\right)\left(1+\mathrm{e}^{-2(\kappa+\chi) t}\right) & (\kappa \neq \chi) \\
\left(\bar{n}+\frac{1}{2}\right)^{2}\left(\frac{1}{2}+\kappa t\right)\left(e^{-4 \kappa t}+1\right) & (\kappa=\chi)
\end{array},\right.
$$

and behaves for $t \rightarrow \infty$ as,

$$
\Delta x^{2}(t) \Delta y^{2}(t) \underset{t \rightarrow \infty}{\sim}\left\{\begin{array}{ll}
\left(\bar{n}+\frac{1}{2}\right)^{2} & (\kappa=0) \\
\left(\bar{n}+\frac{1}{2}\right)^{2} \frac{\kappa \chi}{\chi^{2}-\rho^{2}} \mathrm{e}^{2(\chi-\kappa) t} & (0<\kappa<\chi) \\
\left(\bar{n}+\frac{1}{2}\right)^{2} \kappa t & (\kappa=\chi) \\
\left(\bar{n}+\frac{1}{2}\right)^{2} \frac{\kappa^{2}}{\kappa^{2}-\chi^{2}} & (\kappa>\chi)
\end{array} .\right.
$$

While the pure squeezing $(\kappa=0)$ preserves the uncertainty relation, the dissipation causes change in it. When $0<\kappa<\chi$, the uncertainty relation diverges exponentially with respect to time. Thus, there is a drastic change in the behavior of uncertainty relation when dissipation is added to the squeezing process no matter how small the dissipation is. See Fig. 1(b) for the behavior of the uncertainty relation for the typical values of $\rho=\kappa / \chi$.

\section{Discussion}

In this paper, we applied NETFD to the system of degenerate parametric amplification accompanied by dissipation. The most part of the present analysis 
is based on the operator algebra. For a comparison, we presented an alternative treatment, i.e., mapping the dissipative Schrödinger equation into a partial differential equation of a c-number function, in Appendix C. The treatment is quite parallel to the ones in the density operator method $[2,4]$. In order to solve the partial differential equation, an elaborate use of the properties of the Hermite polynomials is required. In contrast, most computations are carried out within simple operator algebra in the present analysis. This is one technical merit of the present method.

In the model we considered, there is coexistence of the dissipation and the symmetry breaking due to the squeezing. We have shown that, in such a case, we can first treat the pure dissipative case to obtain the vacuum $|W(t)\rangle$ for semi-free field and corresponding annihilation-creation operators $\gamma_{t}^{(W)}, \tilde{\gamma}_{t}^{(W)}, \gamma^{q}$ and $\tilde{\gamma}^{+}$, and then analyze the additional effect of squeezing. We note that it is possible to interchange the treatments of dissipation and squeezing, i.e., we can first treat the pure squeezing and then analyze the additional effect of dissipation. The procedure is quite parallel to that in Sec. 5 .

The pure dissipative time-evolution appears as condensation of $\gamma^{q} \tilde{\gamma}^{+}$-pairs into the vacuum $|0\rangle$, while the breaking of the phase symmetry due to $H_{1}$ of (4.13) appears as condensation of a combination of pairs $\left(\gamma^{q} \gamma^{q}+\tilde{\gamma}^{q} \tilde{\gamma}^{q}\right)$. We see from (7.5) that the change of the number of photons $N(t)-n[n=N(0)]$ and the quarter of the difference of the squared quadrature amplitudes $\Delta(t) / 4=$ $\left[\Delta x^{2}(t)-\Delta y^{2}(t)\right] / 4$ are the order parameters associated, respectively, with the pairs $\gamma^{q} \tilde{\gamma}^{+}$and $\left(\gamma^{q} \gamma^{q}+\tilde{\gamma}^{+} \tilde{\gamma}^{q}\right)$.

As we have seen in Sec. 7 , the dissipative squeezed vacuum $|0(t)\rangle$ can be rearranged into the form that the pairs $\gamma^{q} \tilde{\gamma}^{q}$ and $\left(\gamma^{q} \gamma^{q}+\tilde{\gamma}^{q} \tilde{\gamma}^{q}\right)$ condensing into the purely dissipative vacuum $|W(t)\rangle$ as (7.8). The fact implies that we can split the effect of pure dissipation and the remaining effect in a concise form. Similarly, the effect of pure squeezing and the remaining effect can be split as (7.12).

Note that the expression (7.5) of the dissipative squeezed vacuum can be further exploited, for example, in the analysis of the effect of dissipation in quantum information technology for continuous variables [1]. The operator formalism of NETFD is beneficial to such analysis since it allows us to treat the processes such as the dissipative squeezed vacuum entering a beam splitter or projective measurements on the dissipative squeezed vacuum in a similar manner as quantum mechanics. Such applications will be reported elsewhere in the near future.

The estimate of squeezing obtained in Sec. 8 can be used as the estimate of $\kappa$, the attenuation rate inside the nonlinear media, in experiments, when the rate of squeezing $\chi$ is known. One can measure $\Delta x^{2}(t)$ and $\Delta y^{2}(t)$ varying the value of $\chi$ by changing the amplitude of the pumping mode (with $t$ fixed). Then, the value of $\kappa$ can be estimate by fitting (8.1) and (8.2) to the data. However, note that, in actual optical devices, there are many sources of dissipation other than those at the nonlinear media, such as those at mirrors. The present analysis gives the estimate of the contribution from the dissipation inside the nonlinear 
media.

The effect of dissipation on the uncertainty relation $\Delta x \Delta y$ estimated in Sec. 8 suggests that, when squeezing and dissipation are both present, the relation $\Delta x \Delta y=\bar{n}+1 / 2$ at thermal equilibrium state easily fails to be satisfied no matter how small the dissipation is. Thus, one must be cautious of using $\Delta x \Delta y \sim$ $\bar{n}+1 / 2$ even as a rough estimate.

Finally, note that we have introduced the dissipation and the squeezing independently following the model in the damping theory $[2,4]$, i.e., the hatHamiltonian corresponds to squeezing $\hat{H}_{1}$ is simply added to the semi-free hatHamiltonian $\hat{H}_{0}+\mathrm{i} \hat{\Pi}$ as (4.4). It was pointed out by Celeghini et al. [16] that the dissipation and the squeezing are related to each other in a fundamental aspect. It is of interest to investigate such relation and see whether the hat-Hamiltonian (4.4) should be modified within the framework of NETFD. However, it is out of the scope of the present study.

\section{A The principle of correspondence}

"The principle of correspondence" is defined by $[17,5,6]$,

$$
\begin{aligned}
\rho_{S}(t) & \longleftrightarrow|0(t)\rangle, \\
A_{1} \rho_{S}(t) A_{2} & \longleftrightarrow A_{1} \tilde{A}_{2}^{\dagger}|0(t)\rangle .
\end{aligned}
$$

The quantum master equation in the density operator method and the dissipative Schrödinger equation in NETFD can be related to each other through "the principle of correspondence". It was noticed first by Crawford [18] that, with the introduction of two kinds of operators, the Liouville-von Neumann equation for non-dissipative systems can be handled as the Schrödinger equation.

\section{B Relation between Schrödinger and interaction representations}

In this section, we restore the subscript I that we have dropped since Sec. 5 and see the relation between the expectation values with respect to $|0(t)\rangle$ in the Schrödinger representation and those with respect to $|0(t)\rangle_{\mathrm{I}}$ in the the interaction representation. For arbitrary operator $A$, the expectation values $\langle 1|A| 0(t)\rangle_{\mathrm{I}}$ and $\langle 1|A| 0(t)\rangle$ can be transformed to each other through the relation

$$
\langle 1|A| 0(t)\rangle=\left\langle 1\left|A_{\mathrm{I}}(t)\right| 0(t)\right\rangle_{\mathrm{I}},
$$

where $A_{\mathrm{I}}(t):=\mathrm{e}^{\mathrm{i} \hat{H}_{0} t} A \mathrm{e}^{-\mathrm{i} \hat{H}_{0} t}$. For $A=a, a^{\dagger}$, we have

$$
\begin{aligned}
\langle 1|a| 0(t)\rangle & =\left\langle 1\left|a_{\mathrm{I}}(t)\right| 0(t)\right\rangle_{\mathrm{I}}=\langle 1|a| 0(t)\rangle_{\mathrm{I}} \mathrm{e}^{-\mathrm{i} \omega t}, \\
\left\langle 1\left|a^{\dagger}\right| 0(t)\right\rangle & =\left\langle 1\left|a_{\mathrm{I}}^{\dagger}(t)\right| 0(t)\right\rangle_{\mathrm{I}}=\left\langle 1\left|a^{\dagger}\right| 0(t)\right\rangle_{\mathrm{I}} \mathrm{e}^{\mathrm{i} \omega t}
\end{aligned}
$$


Similarly, we have

$$
\begin{aligned}
x(t)= & x_{\mathrm{I}}(t) \cos \omega t+y_{\mathrm{I}}(t) \sin \omega t, \\
y(t)= & -x_{\mathrm{I}}(t) \sin \omega t+y_{\mathrm{I}}(t) \cos \omega t, \\
\Delta x^{2}(t)= & \Delta x_{\mathrm{I}}^{2}(t) \cos ^{2} \omega t+\Delta y_{\mathrm{I}}^{2}(t) \sin ^{2} \omega t \\
& +\left[2 \Delta x y_{\mathrm{I}}(t)-\mathrm{i}\right] \cos \omega t \sin \omega t, \\
\Delta y^{2}(t)= & \Delta x_{\mathrm{I}}^{2}(t) \sin ^{2} \omega t+\Delta y_{\mathrm{I}}^{2}(t) \cos ^{2} \omega t \\
& -\left[2 \Delta x y_{\mathrm{I}}(t)-\mathrm{i}\right] \cos \omega t \sin \omega t,
\end{aligned}
$$

where

$$
\begin{array}{rlrl}
x(t) & :=\langle 1|q| 0(t)\rangle, & y(t):=\langle 1|p| 0(t)\rangle, \\
\Delta x^{2}(t) & :=\left\langle 1\left|[q-x(t)]^{2}\right| 0(t)\right\rangle, & \Delta y^{2}(t):=\left\langle 1\left|[p-y(t)]^{2}\right| 0(t)\right\rangle, \\
x_{\mathrm{I}}(t) & :=\langle 1|q| 0(t)\rangle_{\mathrm{I}}, & y_{\mathrm{I}}(t):=\langle 1|p| 0(t)\rangle_{\mathrm{I}}, \\
\Delta x_{\mathrm{I}}^{2}(t) & :=\left\langle 1\left|\left[q-x_{\mathrm{I}}(t)\right]^{2}\right| 0(t)\right\rangle_{\mathrm{I}}, & \Delta y_{\mathrm{I}}^{2}(t):=\left\langle 1\left|\left[p-y_{\mathrm{I}}(t)\right]^{2}\right| 0(t)\right\rangle_{\mathrm{I}}, \\
\Delta x y_{\mathrm{I}}(t) & :=\left\langle 1\left|\left[q-x_{\mathrm{I}}(t)\right]\left[p-y_{\mathrm{I}}(t)\right]\right| 0(t)\right\rangle_{\mathrm{I}} .
\end{array}
$$

\section{Treatment in a c-number function space}

In the present study, we exploited the operator algebras within NETFD to handle the dissipative squeezing process. In this section, we show an alternative treatment in NETFD, i.e., we map the dissipative Schrödinger equation into a partial differential equation of a c-number function and then solve the equation. The treatment is quite parallel to the ones in the density operator method $[2$, 4], in which the quantum master equation is mapped to a partial differential equation of a c-number function.

Let us introduce, a set of states $\mid m, n)$ which are simultaneous eigenstates of $a^{\dagger} a$ and $\tilde{a}^{\dagger} \tilde{a}$, i.e.,

$$
\left.\left.\left.a^{\dagger} a \mid m, n\right)=m(m, n), \quad \tilde{a}^{\dagger} \tilde{a} \mid m, n\right)=n \mid m, n\right),
$$

where $m, n$ are 0 or positive integers. The state $\mid 0,0)$ is the vacuum annihilated by $a$ and $\tilde{a}$, i.e.,

$$
a \mid 0,0)=0, \quad \tilde{a} \mid 0,0)=0 .
$$

We assume the ortho-normality and the completeness of $\mid m, n)$, i.e.,

$$
\left.\left(m^{\prime}, n^{\prime} \mid m, n\right)=\delta_{m^{\prime} m} \delta_{n^{\prime} n}, \quad \sum_{m, n} \mid m, n\right)(m, n \mid=1 .
$$

The thermal bra-vacuum $\langle 1|$ can be represented as

$$
\langle 1|=\sum_{n}(n, n) .
$$


We define the coherent state $|z, w\rangle$ by

$$
|z, w\rangle:=D(z) \widetilde{D(w)} \mid 0,0),
$$

with

$$
D(z):=\mathrm{e}^{z a^{\dagger}-z^{*} a},
$$

where $z$ and $w$ are complex numbers. The conjugate bra-state $\langle z, w|$ is given by

$$
\langle z, w|=\left(0,0 \mid D^{\dagger}(z) \widetilde{D^{\dagger}(w)} .\right.
$$

One can easily show that

$$
\begin{aligned}
a|z, w\rangle & =z|z, w\rangle, \quad \tilde{a}|z, w\rangle=w^{*}|z, w\rangle, \\
\langle z, w| a^{\dagger} & =\langle z, w| z^{*}, \quad\langle z, w| \tilde{a}^{\dagger}=\langle z, w| w,
\end{aligned}
$$

and

$$
\int \frac{\mathrm{d}^{2} z}{\pi} \int \frac{\mathrm{d}^{2} w}{\pi}|z, w\rangle\langle z, w|=1,
$$

where $\mathrm{d}^{2} z:=\mathrm{d}(\operatorname{Re} z) \mathrm{d}(\operatorname{Im} z)$ and $\mathrm{d}^{2} w:=\mathrm{d}(\operatorname{Re} w) \mathrm{d}(\operatorname{Im} w)$.

By using the coherent states, the vacuum $|0(t)\rangle$ can be mapped to a c-number function by

$$
Q\left(z, z^{*}, t\right)=\frac{1}{\pi}\langle z, z \mid 0(t)\rangle .
$$

The function $Q\left(z, z^{*}, t\right)$ is identical to the $\mathrm{Q}$ function in the density operator method [3]. Note that, we have

$$
\begin{aligned}
\left\langle 1\left|a^{m} a^{\dagger^{n}}\right| 0(t)\right\rangle & =\left\langle 1\left|a^{m} \int \frac{\mathrm{d}^{2} z}{\pi} \int \frac{\mathrm{d}^{2} w}{\pi}\right| z, w\right\rangle\left\langle z, w\left|a^{\dagger^{n}}\right| 0(t)\right\rangle \\
& =\int \frac{\mathrm{d}^{2} z}{\pi} \int \frac{\mathrm{d}^{2} w}{\pi} z^{m} z^{* n}\langle 1 \mid z, w\rangle\langle z, w \mid 0(t)\rangle .
\end{aligned}
$$

After some computation with the use of the identity

$$
\mathrm{e}^{A} \mathrm{e}^{B}=\mathrm{e}^{A+B} \mathrm{e}^{\frac{1}{2}[A, B]} \quad(\text { for }[[A, B], A]=[[A, B], B]=0),
$$

we obtain,

$$
\begin{aligned}
\langle 1 \mid z, w\rangle & =\mathrm{e}^{\frac{1}{2}|z-w|^{2}} \mathrm{e}^{\frac{1}{2}\left(z w^{*}-z^{*} w\right)}, \\
\langle z, w| & =\langle z, z|[D(z-w)]^{\sim} \mathrm{e}^{\frac{1}{2}\left(z^{*} w-z w^{*}\right)}, \\
\int \frac{\mathrm{d}^{2} z}{\pi} \mathrm{e}^{-\frac{|z|^{2}}{2}} D(z) & =1,
\end{aligned}
$$

where the thermal state condition (2.6) is used in deriving (C.14). From (C.12)(C.16), we have

$$
\left\langle 1\left|a^{m} a^{\dagger^{n}}\right| 0(t)\right\rangle=\int \mathrm{d}^{2} z z^{m} z^{* n} Q\left(z, z^{*}, t\right) .
$$


Hence, for a function $f\left(a, a^{\dagger}\right)$ of the operators given in the anti-normal ordered form, the expectation value is given in terms of $Q\left(z, z^{*}, t\right)$ by

$$
\left\langle 1\left|f\left(a, a^{\dagger}\right)\right| 0(t)\right\rangle=\int \mathrm{d}^{2} z f\left(z, z^{*}\right) Q\left(z, z^{*}, t\right) .
$$

By taking the inner product of $\langle z, z|$ and the dissipative Schrödinger equation (5.1) for the dissipative squeezed vacuum, we obtain the partial differential equation for $Q\left(z, z^{*}, t\right)$ as

$$
\begin{aligned}
\frac{\partial}{\partial t} Q\left(z, z^{*}, t\right)=\{ & -\chi\left[\frac{\partial}{\partial z^{*}} z+\frac{\partial}{\partial z} z^{*}+\frac{1}{2}\left(\frac{\partial^{2}}{\partial z^{2}}+\frac{\partial^{2}}{\partial z^{* 2}}\right)\right] \\
& \left.+\kappa\left[\frac{\partial}{\partial z} z+\frac{\partial}{\partial z^{*}} z^{*}+2(\bar{n}+1) \frac{\partial}{\partial z} \frac{\partial}{\partial z^{*}}\right]\right\} Q\left(z, z^{*}, t\right),
\end{aligned}
$$

where we have used the identities

$$
\langle z, z| a=\left(z+\frac{\partial}{\partial z^{*}}\right)\langle z, z|, \quad\langle z, z| \tilde{a}=\left(z^{*}+\frac{\partial}{\partial z}\right)\langle z, z| .
$$

By introducing the new variables $x$ and $y$ as

$$
\begin{gathered}
x=\frac{z+z^{*}}{\sqrt{2}}, \quad y=\frac{z-z^{*}}{\sqrt{2} \mathrm{i}}, \\
Q(x, y, t) \mathrm{d} x \mathrm{~d} y=Q\left(z, z^{*}, t\right) \mathrm{d}^{2} z,
\end{gathered}
$$

(C.19) reduces to

$$
\begin{aligned}
\frac{\partial}{\partial t} Q(x, y, t)=\{ & {\left[(-\chi+\kappa) \frac{\partial}{\partial x} x+\left(-\frac{\chi}{2}+\kappa(\bar{n}+1)\right) \frac{\partial^{2}}{\partial x^{2}}\right] } \\
& \left.+\left[(\chi+\kappa) \frac{\partial}{\partial y} y+\left(\frac{\chi}{2}+\kappa(\bar{n}+1)\right) \frac{\partial^{2}}{\partial y^{2}}\right]\right\} Q(x, y, t) .
\end{aligned}
$$

Suppose the solution of (C.23) is given by the form

$$
Q(x, y, t)=f_{+}(x) f_{-}(y) g(t) .
$$

Then, $f_{ \pm}(x)$ and $g(t)$ satisfy the ordinary differential equations

$$
\begin{array}{r}
{\left[\left(\mp \frac{\chi}{2}+\kappa(\bar{n}+1)\right) \frac{\mathrm{d}^{2}}{\mathrm{~d} x^{2}}+(\mp \chi+\kappa) \frac{\mathrm{d}}{\mathrm{d} x} x-\lambda_{ \pm}\right] f_{ \pm}(x)=0,} \\
\left(\frac{\mathrm{d}}{\mathrm{d} t}-\lambda_{g}\right) g(t)=0,
\end{array}
$$

where $\lambda_{g}=\lambda_{+}+\lambda_{-}$. Note that the differential equation

$$
\left[\frac{\mathrm{d}^{2}}{\mathrm{~d} x^{2}}+A \frac{\mathrm{d}}{\mathrm{d} x} x+B\right] f(x)=0
$$


has the solutions

$$
f(x)= \begin{cases}H_{m}\left(\sqrt{\frac{A}{2}} x\right) \mathrm{e}^{-\frac{A x^{2}}{2}} \quad\left(m=\frac{B}{A}\right) & \text { for } A>0 \\ H_{m}\left(\sqrt{\left.\frac{|A|}{2} x\right)} \quad\left(m=\frac{B-|A|}{|A|}\right)\right. & \text { for } A<0, \\ C_{1} \mathrm{e}^{\sqrt{-B} x}+C_{2} \mathrm{e}^{-\sqrt{-B} x} & \text { for } A=0\end{cases}
$$

where $m$ is 0 or a positive integer, $C_{1}$ and $C_{2}$ are constants, and $H_{m}(x)$ is the Hermite polynomial defined by

$$
H_{m}(x):=(-1)^{n} \mathrm{e}^{x^{2}} \frac{\mathrm{d}^{n}}{\mathrm{~d} x^{n}} \mathrm{e}^{-x^{2}} .
$$

Note also that the differential equation

$$
\left[\frac{\mathrm{d}}{\mathrm{d} x} x+B\right] f(x)=0,
$$

has the solution

$$
f(x)=C x^{-B-1},
$$

where $C$ is a constant.

By noting (C.28) and (C.31), we find that the general solution of (C.23) is given as follows. Hereafter, without loss of generality, we assume $\chi \geq 0$.

(i) $0 \leq \chi<\kappa, \chi>2 \kappa(\bar{n}+1)$

$$
\begin{aligned}
& Q(x, y, t)=\sum_{m_{+}, m_{-}=0}^{\infty}\left[C_{m_{+}, m_{-}} H_{m_{+}}\left(\sqrt{\frac{A_{+}}{2}} x\right) \exp \left(-\frac{A_{+} x^{2}}{2}\right)\right. \\
& \times H_{m_{-}}\left(\sqrt{\frac{A_{-}}{2} y}\right) \exp \left(-\frac{A_{-} y^{2}}{2}\right) \\
& \left.\times \exp \left\{\left[m_{+}(\chi-\kappa)+m_{-}(-\chi-\kappa)\right] t\right\}\right] \text {, }
\end{aligned}
$$

(ii) $\kappa<\chi<2 \kappa(\bar{n}+1)$

$$
\begin{aligned}
Q(x, y, t)=\sum_{m_{+}, m_{-}=0}^{\infty}[ & C_{m_{+}, m_{-}} H_{m_{+}}\left(\sqrt{\frac{\left|A_{+}\right|}{2}} x\right) \\
& \times H_{m_{-}}\left(\sqrt{\frac{A_{-}}{2}} y\right) \exp \left(-\frac{A_{-} y^{2}}{2}\right) \\
& \left.\times \exp \left\{\left[\left(m_{+}+1\right)(-\chi+\kappa)+m_{-}(-\chi-\kappa)\right] t\right\}\right],
\end{aligned}
$$


(iii) $\chi=\kappa$

$$
\begin{aligned}
& Q(x, y, t)=\int_{-\infty}^{\infty} \mathrm{d} k \sum_{m_{-}=0}^{\infty}\left[C_{k, m_{-}} \exp (\mathrm{i} k x)\right. \\
& \times H_{m_{-}}\left(\sqrt{\frac{A_{-}}{2}} y\right) \exp \left(-\frac{A_{-} y^{2}}{2}\right) \\
& \left.\times \exp \left\{\left[-\left(\bar{n}+\frac{1}{2}\right) k^{2}-2 m_{-}\right] \kappa t\right\}\right] \text {, }
\end{aligned}
$$

(iv) $\chi=2 \kappa(\bar{n}+1)$

$$
\begin{aligned}
& Q(x, y, t)=\sum_{m_{+}, m_{-}=0}^{\infty}\left[C_{m_{+}, m_{-}} x^{m_{+}}\right. \\
& \times H_{m_{-}}\left(\sqrt{\frac{A_{-}}{2} y}\right) \exp \left(-\frac{A_{-} y^{2}}{2}\right) \\
& \left.\times \exp \left\{\left[-\left(m_{+}+1\right)(2 \bar{n}+1)-2 m_{-} \bar{n}\right] \kappa t\right\}\right] \text {, }
\end{aligned}
$$

where

$$
A_{ \pm}:=\frac{ \pm \chi+\kappa}{ \pm \frac{\chi}{2}+\kappa(\bar{n}+1)},
$$

$C_{m_{+}, m_{-}}$and $C_{k, m_{-}}$are constants.

Let the system be initially $(t=0)$ at thermal equilibrium state with temperature $T_{0}$. Then, $Q(x, y, t=0)$ is given by

$$
Q(x, y, t=0)=\frac{1}{2 \pi(n+1)} \exp \left[-\frac{x^{2}+y^{2}}{2(n+1)}\right],
$$

where $n$ is the number of the photons given by (3.18). Since $Q(x, y, t=0)$ can be written in a form of product $Q_{+}(x) Q_{-}(y)$, the coefficients $C_{m_{+}, m_{-}}\left(C_{k, m_{-}}\right)$ also have a form of product,

$$
C_{m_{+}, m_{-}}=C_{m_{+}} C_{m_{-}} \quad\left(C_{k, m_{-}}=C_{k} C_{m_{-}}\right) .
$$

It follows that $Q(x, y, t)$ at any time $t>0$ can be written in a form of product

$$
Q(x, y, t)=Q_{+}(x, t) Q_{-}(y, t),
$$

where $Q_{ \pm}(x, t)$ will be specified for individual cases (i)-(iv) as follows. 
$\frac{(i) 0 \leq \chi<\kappa, \chi>2 \kappa(\bar{n}+1)}{Q_{ \pm}(x, t) \text { are given by }}$

$$
Q_{ \pm}(x, t)=\sum_{m_{ \pm}=0}^{\infty} C_{m_{ \pm}} H_{m_{ \pm}}\left(\sqrt{\frac{A_{ \pm}}{2}} x\right) \exp \left(-\frac{A_{ \pm} x^{2}}{2}\right) \exp \left[m_{ \pm}( \pm \chi-\kappa) t\right] .
$$

Let us expand the Gaussian distribution function with the mean 0 and the variance $\sigma$ as follows,

$$
\frac{1}{\sqrt{2 \pi \sigma}} \exp \left(-\frac{x^{2}}{2 \sigma}\right)=\sum_{m=0}^{\infty} G_{m}(\sigma, A) H_{m}\left(\sqrt{\frac{A}{2}} x\right) \exp \left(-\frac{A x^{2}}{2}\right),
$$

where $G_{m}(\sigma, A)$ are the expansion coefficients. They are obtained as

$$
G_{m}(\sigma, A)= \begin{cases}\frac{1}{2^{m}(m / 2) ! \sqrt{\pi}} \frac{A}{2}(A \sigma-1)^{m / 2} & (m: \text { even }) \\ 0 & (m: \text { odd })\end{cases}
$$

by using the properties of Hermitian polynomials,

$$
\begin{aligned}
\int_{-\infty}^{\infty} \mathrm{d} x H_{m}(x) H_{l}(x) e^{-x^{2}} & =m ! 2^{m} \sqrt{\pi} \delta_{m l}, \\
\int_{-\infty}^{\infty} \mathrm{d} x \exp \left(-a x^{2}\right) H_{m}(x) & = \begin{cases}\frac{m !}{(m / 2) !} \sqrt{\frac{\pi}{a}}\left(\frac{1}{a}-1\right)^{m / 2} & (m: \text { even }) \\
0 & (m: \text { odd })\end{cases}
\end{aligned}
$$

From (C.37), (C.40) and (C.41), we find that

$$
C_{m_{ \pm}}=G_{m_{ \pm}}\left(n+1, A_{ \pm}\right),
$$

and that

$$
\begin{aligned}
Q_{ \pm}(x, t)= & \sum_{m_{ \pm}=0}^{\infty} G_{m_{ \pm}}\left(n+1, A_{ \pm}\right) H_{m_{ \pm}}\left(\sqrt{\frac{A_{ \pm}}{2}} x\right) \exp \left(-\frac{A_{ \pm} x^{2}}{2}\right) \\
& \quad \times \exp \left[m_{ \pm}( \pm \chi-\kappa) t\right] \\
= & \sum_{m_{ \pm}=0}^{\infty} G_{m_{ \pm}}\left(\sigma_{ \pm}(t), A_{ \pm}\right) H_{m_{ \pm}}\left(\sqrt{\frac{A_{ \pm}}{2}} x\right) \exp \left(-\frac{A_{ \pm} x^{2}}{2}\right) \\
= & \frac{1}{\sqrt{2 \pi \sigma_{ \pm}(t)}} \exp \left(-\frac{x^{2}}{2 \sigma_{ \pm}(t)}\right)
\end{aligned}
$$

with

$$
\sigma_{ \pm}(t)=n+1+\frac{ \pm \chi\left(\bar{n}+\frac{1}{2}\right)-\kappa(n-\bar{n})}{\kappa \mp \chi}\left(1-\mathrm{e}^{-2(\kappa \pm \chi) t}\right) .
$$


$\frac{\text { (ii) } \kappa<\chi<2 \kappa(\bar{n}+1)}{Q_{+}(x, t) \text { is }}$

$$
Q_{+}(x, t)=\sum_{m_{+}=0}^{\infty} C_{m_{+}} H_{m_{+}}\left(\sqrt{\frac{\left|A_{+}\right|}{2} x}\right) \exp \left[m_{+}( \pm \chi-\kappa) t\right] .
$$

Let us introduce another expansion of the Gaussian distribution function,

$$
\frac{1}{\sqrt{2 \pi \sigma}} \exp \left(-\frac{x^{2}}{2 \sigma}\right)=\sum_{m=0}^{\infty} G_{m}^{\prime}(\sigma, A) H_{m}\left(\sqrt{\frac{|A|}{2} x}\right),
$$

where $G_{m}^{\prime}(\sigma, A)$ is given by

$$
G_{m}^{\prime}(\sigma, A)=-\frac{1}{2^{m}(m / 2) !} \sqrt{\frac{|A|}{2 \pi}}(-1)^{m / 2}(1+\sigma|A|)^{-(m+1) / 2} .
$$

From (C.37), (C.48) and (C.49), we find that

$$
C_{m_{+}}=G_{m_{+}}^{\prime}\left(n+1, A_{+}\right),
$$

and that

$$
\begin{aligned}
Q_{+}(x, t) & =\sum_{m_{+}} G_{m_{+}}^{\prime}\left(n+1, A_{+}\right) H_{m_{+}}\left(\sqrt{\frac{|A|}{2}}\right) \exp \left[\left(m_{+}+1\right)(-\chi-\kappa) t\right] \\
& =\sum_{m_{+}} G_{m_{+}}^{\prime}\left(\sigma_{+}(t), A_{+}\right) H_{m_{+}}\left(\sqrt{\frac{|A|}{2}}\right) \\
& =\frac{1}{\sqrt{2 \pi \sigma_{+}(t)}} \exp \left(-\frac{x^{2}}{2 \sigma_{+}(t)}\right),
\end{aligned}
$$

with $\sigma_{+}(t)$ of (C.47). $Q_{-}(y, t)$ is obtained as that in (C.46) in the same way as for (i).

(iii) $\chi=\kappa$

$\overline{Q_{+}(x, t)}$ is given by

$$
Q_{+}(x, t)=\int_{-\infty}^{\infty} \mathrm{d} k C_{k} \exp (\mathrm{i} k x) \exp \left[-\left(\bar{n}+\frac{1}{2}\right) \kappa k^{2} t\right]
$$

From (C.37) and (C.53), we find

$$
C_{k}=\frac{1}{2 \pi} \exp \left(-\frac{\bar{n}+1}{2} k^{2}\right)
$$

and that

$$
Q_{+}(x, t)=\frac{1}{\sqrt{2 \pi \sigma_{+}(t)}} \exp \left(-\frac{x^{2}}{2 \sigma_{+}(t)}\right)
$$


with

$$
\sigma_{+}(t)=\bar{n}+1+(2 \bar{n}+1) \kappa t .
$$

$Q_{-}(y, t)$ is obtained as that in (C.46) in the same way as for (i).

(iv) $\chi=2 \kappa(\bar{n}+1)$

$\overline{Q_{+}(x, t)}$ is given by

$$
Q_{+}(x, t)=\sum_{m_{+}=0}^{\infty} C_{m_{+}} x^{m_{+}} \exp \left[-\left(m_{+}+1\right) \kappa(2 \bar{n}+1) t\right] .
$$

From (C.37) and (C.57), we find

$$
C_{m_{+}}= \begin{cases}\frac{1}{\sqrt{2 \pi(n+1)}} \frac{1}{\left(m_{+} / 2\right) !}\left(-\frac{1}{2(n+1)}\right)^{m_{+} / 2} & \left(m_{+}: \text {even }\right) \\ 0 & \left(m_{+}: \text {odd }\right)\end{cases}
$$

and that

$$
\begin{aligned}
Q_{+}(x, t)= & \frac{1}{\sqrt{2 \pi(n+1)}} \\
& \times \sum_{m^{\prime}=0}^{\infty} \frac{1}{m^{\prime} !}\left(-\frac{1}{2(n+1)}\right)^{m^{\prime}} x^{2 m^{\prime}} \exp \left[-\left(2 m^{\prime}+1\right) \kappa(2 \bar{n}+1) t\right] \\
= & \frac{1}{\sqrt{2 \pi \sigma_{+}(t)}} \exp \left(-\frac{x^{2}}{2 \sigma(t)}\right),
\end{aligned}
$$

with

$$
\sigma_{+}(t)=(n+1) \mathrm{e}^{2 \kappa(2 \bar{n}+1) t} .
$$

$Q_{-}(y, t)$ is obtained as that in (C.47) in the same way as for (i).

To summarize, $Q(x, y, t)$ is given as

$$
Q(x, y, t)=\frac{1}{2 \pi \sqrt{\sigma_{+}(t) \sigma_{-}(t)}} \exp \left[-\frac{x^{2}}{2 \sigma_{+}(t)}-\frac{y^{2}}{2 \sigma_{-}(t)}\right],
$$

where $\sigma_{ \pm}(t)$ is given by (C.47) and $\sigma_{+}(t)$ for $\chi=\kappa$ should be interpreted as the limiting value for $\chi \rightarrow \kappa$. Now, $Q\left(z, z^{*}, t\right)$ reads

$$
Q\left(z, z^{*}, t\right)=\frac{1}{\pi \sqrt{\sigma_{+}(t) \sigma_{-}(t)}} \exp \left[-\frac{(\operatorname{Re} z)^{2}}{\sigma_{+}(t)}-\frac{(\operatorname{Im} z)^{2}}{\sigma_{-}(t)}\right],
$$


and the expectation values of quadrature amplitudes are given by

$$
\begin{aligned}
x(t) & :=\int \mathrm{d}^{2} z Q\left(z, z^{*}\right) \frac{z+z^{*}}{\sqrt{2}}=0, \\
y(t) & :=\int \mathrm{d}^{2} z Q\left(z, z^{*}\right) \frac{z-z^{*}}{\sqrt{2} \mathrm{i}}=0, \\
\Delta x^{2}(t) & =\frac{1}{2} \int \mathrm{d}^{2} z Q\left(z, z^{*}\right)\left(z^{2}+2 z z^{*}-1+z^{* 2}\right), \\
& =\sigma_{+}(t)-\frac{1}{2}, \\
\Delta y^{2}(t) & =\frac{1}{2} \int \mathrm{d}^{2} z Q\left(z, z^{*}\right)\left(-z^{2}+2 z z^{*}-1-z^{* 2}\right), \\
& =\sigma_{-}(t)-\frac{1}{2} .
\end{aligned}
$$

We see that (C.63)-(C.66) with (C.47) are consistent with (6.6) and (6.7).

\section{References}

[1] A. Furusawa, J. L. Sørensen, C. Braunstein, C. A. Fuchs, H. J. Kimble, E. S. Polzik, Science 282(1998) 706-708.

[2] F. Haake, Springer Tracts Mod. Phys. 66(1973) 98-168.

[3] G.S. Agarwal, E. Wolf, Phys. Rev. D 2(1970) 2161-2186; ibid. 2187-2205; ibid. 2206-2225.

[4] M. T. Raiford, Phys. Rev. A 2(1970) 1541-1558.

[5] T. Arimitsu and H. Umezawa. Prog. Theor. Phys. 74(1985) 429-432.

[6] T. Arimitsu and H. Umezawa. Prog. Theor. Phys. 77(1987) 32-52.

[7] T. Arimitsu and H. Umezawa. Prog. Theor. Phys. 77(1987) 53-67.

[8] T. Hayashi, Application of Non-Equilibrium Thermo Field Dynamics to Non-Abelian Gauge Theory and to Quantum Information Theory, PhD thesis, Grad. Schl. of Pure and Applied Sciences, Univ. of Tsukuba, 2006. Appendix A.

[9] T. Arimitsu, Condens. Matter Phys. 4(1994) 26-88. Available from http://www.px.tsukuba.ac.jp/home/tcm/arimitsu/cmp4.pdf.

[10] A.E. Kobryn, T. Hayashi, and T. Arimitsu, Ann. Phys. 308(2003) 395446.

[11] L. Leplae, H. Umezawa, and F. Mancini, Physics Reports 10C(1974) 151272 . 
[12] Y. Takahashi and H. Umezawa, Collect. Phenom. 2(1975) 55-80.

[13] T. Arimitsu, Y. Sudo, and H. Umezawa, Physica A 146(1987) 433-451.

[14] K. Yoshida and T. Arimitsu, Condens. Matter Phys. 11(2008) 687-697.

[15] E. Celeghini, M. Rasetti, and G. Vitiello. Annals of Physics 215(1992) 156-170.

[16] E. Celeghini, M. Rasetti, M. Tarlini, and G. Vitiello, Mod. Phys. Lett. B 3(1989) 1213-1220.

[17] M. Schmutz, Z. Phys. B 30(1978) 97-106.

[18] J.A. Crawford, Nuovo Cim. 10(1958) 698-713. 